\title{
Experimental validation of RANS and DES modelling of pipe flow mixing
}

\author{
Gotfredsen, Erik; Kunoy, Jens Dahl; Mayer, Stefan; Meyer, Knud Erik
}

Published in:

Heat and Mass Transfer

Link to article, DOI:

10.1007/s00231-020-02835-8

Publication date:

2020

Document Version

Peer reviewed version

Link back to DTU Orbit

Citation (APA):

Gotfredsen, E., Kunoy, J. D., Mayer, S., \& Meyer, K. E. (2020). Experimental validation of RANS and DES modelling of pipe flow mixing. Heat and Mass Transfer, 56, 2211-2224. https://doi.org/10.1007/s00231-02002835-8

\section{General rights}

Copyright and moral rights for the publications made accessible in the public portal are retained by the authors and/or other copyright owners and it is a condition of accessing publications that users recognise and abide by the legal requirements associated with these rights.

- Users may download and print one copy of any publication from the public portal for the purpose of private study or research.

- You may not further distribute the material or use it for any profit-making activity or commercial gain

- You may freely distribute the URL identifying the publication in the public portal

If you believe that this document breaches copyright please contact us providing details, and we will remove access to the work immediately and investigate your claim. 


\title{
Experimental Validation of RANS and DES Modelling of Pipe Flow Mixing
}

\author{
Erik Gotfredsen · Jens Dahl Kunoy · Stefan Mayer · Knud Erik Meyer
}

Received: date / Accepted: date

\begin{abstract}
Velocity and concentration profiles in a pipe flow was measured downstream of injection of a tracer gas at the pipe centerline. The pipe had diameter $D=0.2 \mathrm{~m}$ and two Reynolds numbers, $\operatorname{Re}=50000$ and $\operatorname{Re}=100000$, were used. The profiles were measured at positions $5 D$ and $10 D$ downstream of the injection point. Three different industrial relevant geometrical configurations were used upstream of the injection point: a $10 \mathrm{D}$ straight pipe, two $10 \mathrm{D}$ pipes connected with a $90^{\circ}$ bend or a straight $10 D$ pipe with a mixer plate mounted $2 D$ upstream the injection point. In all cases, air entered the pipe from the surroundings through a sharpedged inlet. This represents many practical flow applications and is also a well-defined inlet condition that generates turbulence in the vena contracta in the inlet. The measurements were compared to predictions from three different computational models: two with Reynolds Averaged Navier-Stokes (RANS) and one with high-resolution Detached Eddy Simulation (DES). For RANS, the k- $\omega$ SST model had difficulty in predicting the turbulence created by the vena contracta. The k- $\varepsilon$ model performed better, but gave completely wrong results for the inlet with a pipe bend. The DES was successful for all cases with only minor deviations from measurements.
\end{abstract}

Keywords Velocity/Concentration measurements · kepsilon model · Scale-Resolving CFD · Hybrid RANS-LES

E. Gotfredsen and K. E. Meyer

Technical University of Denmark

Department of Mechanical Engineering

DK-2800 Kongens Lyngby

Denmark

E-mail: kem@mek.dtu.dk

J. D. Kunoy and S. Mayer

MAN Energy Solution

Copenhagen

Denmark

\section{Introduction}

Studies of the mixing of fluids in pipes have been used to understand the physics of fluids for decades. The probably most famous mixing experiment was made by Osborne Reynolds in 1880 [1]. Different regimes of fluid flow were experimentally investigated by examining mixing of injected ink into water in a pipe flow. In the 1920s, G. I. Taylor [2] investigated the different scales of the flow and made simple mixing correlations. A great deal of research since then has attempted to understand turbulence in order to predict pipeline mixing behaviour. Dimotakis [3] discuss how experiments and simulations become increasingly challenging as the Reynolds number increases. Paul et al [4] summarize that for problems of practical interest, the physics of turbulence cannot presently be solved. The authors also conclude that current models that can be solved, are missing important physics to get accurate simulation of the mixing.

The first attempts to predict mixing used different sophisticated theory-based empirical relations, both for mixing in axial and radial direction. These models were derived and validated with simple experiments, [5] [6] [7] [8] [9] [10]. In the mentioned works, concentration at several positions is investigate in fully develop turbulent pipe flow for high Reynolds numbers $\left(10^{5}\right)$ with point based measurement techniques. A problem with these models is that they are very case specific and, as Clayton's experiments [9] show, hard to validate. Clayton's experiments were performed with a centreline injection into a pipe with Reynolds number at 77000 with the flow being fully turbulent before the injection point. His results show that axial symmetry is hard to obtain, even though the analytical model, assuming Reynolds' analogy, did achieve quite good agreement with the experimental results. Newer studies for examble by Guiraud et al [11] used non-intrusive measuring techniques and still did not get axisymmetric results. All these 
approaches has two premises: the flow must have fully developed turbulence and the geometry of the system must be perfectly symmetric. Both are difficult to obtain experimentally. Paul et al [4] discuss Computational Fluid Dynamics (CFD) models used for prediction of flow mixing. The classical standard Reynolds Averaged Navier-Stokes (RANS) type models typically rely on considerable parameter fitting to give satisfactory mixing results. Detached Eddy Simulation (DES) type models holds the promise of being less dependent on parameter tuning and thus closer to producing trustworthy results "out-of-the-box".

The velocity profile in turbulent pipe flow is well documented, e.g. by Laufer [12]. It has also been demonstrated that Direct Numerical Simulation (DNS) give good agreement with measurements at relatively low Reynolds numbers, e.g. by Eggels et al. [13]. But apparently, very few experiments with detailed measurements of both velocity and concentration in pipe flow are reported in the literature. The present study therefore aims to present accurate measurements of local velocity and concentration for three pipe configurations. In contrast to many earlier studies, the pipe flow is not fully developed. Instead, a 10 diameter long pipe with a sharp-edged inlet is used. The pipe draw air from a larger surrounding space. The sharp edge trigger the creation of a vena contracta that is the main contributor to turbulence. This is a more realistic inlet for industrial applications, where the pipe inlet is often connected to a larger flow container. Also, this inlet conditions do not need to specify measured variables at the inlet. It is interesting to note that designers of RANS models typically have used the fully developed pipe flow as one of their test cases while deciding model parameters. The experiments therefore also offer data that can be used to investigate the turbulence models ability to model pipe flow with more complicated physics in the generation of turbulence.

The three pipe configurations are a straight pipe, a pipe with an upstream $90^{\circ}$ bend and a straight pipe with a mixer plate inserted. These configurations are inspired by actual designs used in large marine diesel engines in the emission control system, but are simplified to be more generic and straightforward to implement in CFD simulations. The configurations also represent application of mixing in a much wider range of process equipment. A tracer gas is injected in the pipe at the centerline, modelling the injection of a reactant gas used in the downstream catalytic reactor. Two different Reynolds numbers of 50000 and 100000 are used to investigate dependence of the Reynolds number. Finally, the pipe configurations are simulated with three fundamentally different turbulence models: two classical RANS models and a computationally more demanding DES model.

\section{Experiment}

\subsection{Setup}

The test rig was based on a straight empty pipe with an inner diameter of $D=200 \mathrm{~mm}$. The pipe was a folded spiralseam pipe with a surface roughness of $25 \mu \mathrm{m}$ estimated from pressure drop measurements. This corresponds to an almost smooth pipe. The pipe had a sharp-edged inlet with a wall thickness of $0.5 \mathrm{~mm}$. The experiments were conducted with ambient air at laboratory conditions. Two flow rates were used matched to Reynolds numbers $\operatorname{Re}_{D}=50000$ and $\operatorname{Re}_{D}=$ 100000. The Reynolds number was based on the pipe diameter and the mean velocity in the pipe found from volume flow measured by an orifice flow meter [14]. The flow meter was placed more than 40 pipe diameters downstream of the test section.

Measurements were obtained with three different configurations in which the conditions upstream of the tracer gas injection position were changed. The three configurations are illustrated in Figure 1. The 'Straight' configuration was an empty pipe with a length of 10D upstream of the injection point. The 'Bend' configuration used a $90^{\circ}$ pipe bend with a radius of curvature of $D$ joined to the upstream end of the 'Straight' configuration. The bend is in the horizontal plane, negative $x$-direction. The bend was connected in upstream direction to a short pipe with a length of $10 D$ and a sharp edged inlet. The 'Plate' configuration consisted of a mixer plate mounted inside the 'Straight' configuration $2 D$ upstream of the injection point. The mixer plate had a diameter $0.5 \mathrm{D}$, a thickness of $3 \mathrm{~mm}$ and was mounted on a horizontal rod. The rod was mounted so that is passed through the pipe center axis. The rod had a diameter of $6 \mathrm{~mm}$. The plate was tilted $15^{\circ}$ from vertical, so that the top was further downstream than the bottom.

For each configuration, velocity and concentration measurements were made along four different lines that crossed the pipe center axis, horizontally or vertically at $\mathrm{z}=5 \mathrm{D}$ and $\mathrm{z}=10 \mathrm{D}$, respectively. They are denoted as $05 \mathrm{DH}, 05 \mathrm{DV}$, $10 \mathrm{DH}$ and 10DV, see Figure 1. Each combination of configuration and position were measured independently. For the measurements of concentration, the tracer gas, methane, was injected at the center line with an injector pipe. The shape of the injector pipe was designed to minimize the effect on the flow in the main pipe and to minimize the level of turbulence in the injected methane. The injector pipe had an outer diameter of $6 \mathrm{~mm}$ and an inner diameter of $3.6 \mathrm{~mm}$. The injector pipe had a $90^{\circ}$ bend with a radius of curvature of $15 \mathrm{~mm}$ followed by a straight part of $36 \mathrm{~mm}$. A HovaGAS Digital G6 flow regulator was used to control and monitor the flow of methane, which was fixed at $Q_{\mathrm{CH}_{4}}=500 \pm 3.6 \frac{\mathrm{ml}}{\mathrm{min}}$. The flow inside the injector is laminar. Note that the mean velocity of the methane at the injection point is about $0.8 \mathrm{~m} / \mathrm{s}$ 


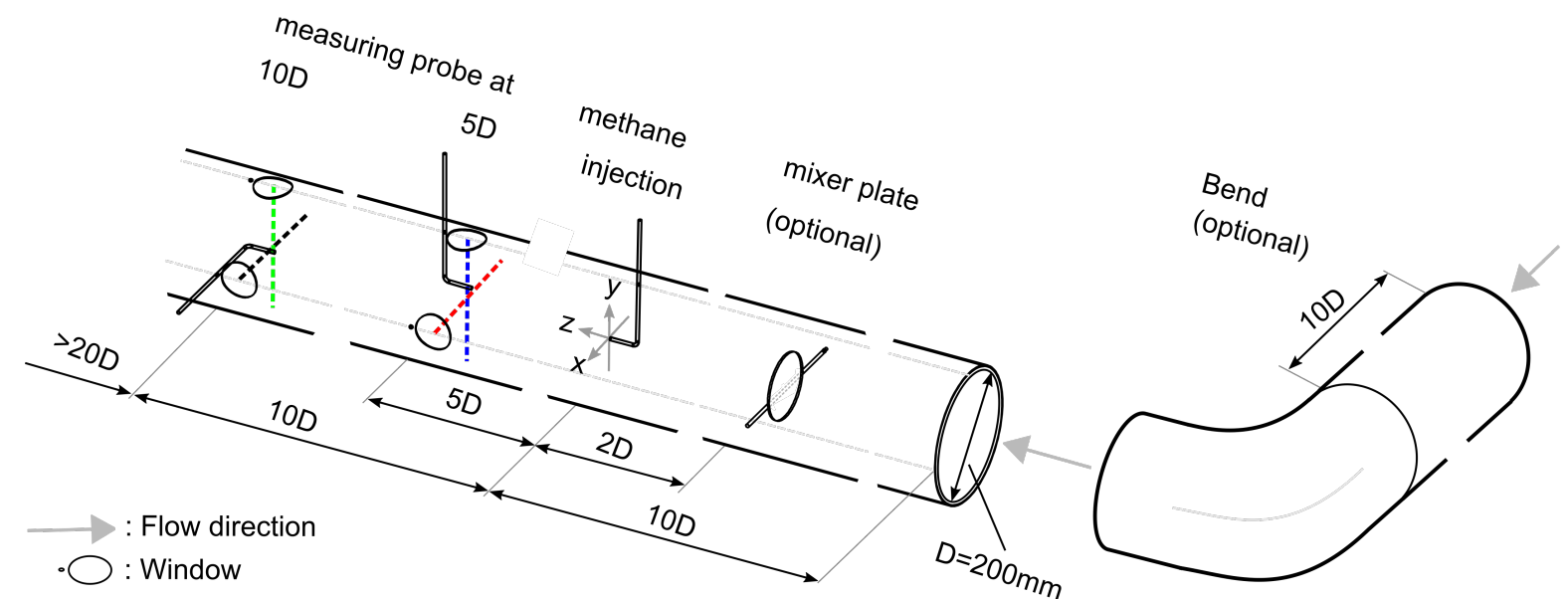

Fig. 1 Sketch of experimental setup where the 'Bend' and 'Plate' are optional. The sketch shows main dimensions, sample positions (coloured dashed lines denoted (---) 05DH, (-- ) 05DV, (-- ) 10DH and (--) 10DV) and the fixed location of the methane injection pipe. Optical access is through two sets of perpendicular $0.1 \mathrm{~m}$ diameter holes covered by a very thin transparent plastic film

while the velocity of the surrounding air is about $4 \mathrm{~m} / \mathrm{s}$. The methane injection and the injection pipe introduce a small wake effect on the pipe flow that only changes the velocity field slightly. The concentration measuring probe had the same dimensions as the injector pipe, but was directed in upstream direction.

\subsection{Measuring techniques}

The longitudinal velocity component $W$ was measured with a Laser Doppler Anemometer (LDA) from Dantec Dynamics. The velocity measurements were made without the probe for measuring methane, but with the injection pipe inserted. Holes with a diameter of $90 \mathrm{~mm}$ were made in the straight pipe and covered with a thin plastic film. A BSA enhanced processor and a Coherent Genesis MX 514-1000 SLM laser, with a wavelengths of $514 \mathrm{~nm}$ and power output set to 30 $\mathrm{mW}$ were used. The optics consisted of a 1.98 beam expander and a $310 \mathrm{~mm}$ focal lens. The angle between the two laser beams was $13.83^{\circ} \pm 0.08^{\circ}$. The beams created a measuring volume with length $630 \mu \mathrm{m}$ and diameter $76 \mu \mathrm{m}$. The software used to collect and process the data was Flow Software Version 2.12.00.15.

The optics were manually aligned perpendicular to the pipe center axis with an uncertainty of $\pm 0.1^{\circ}$ and offset from the centerline of less than $0.5 \mathrm{~mm}$. Glycerine droplets with a diameter of $2 \mu \mathrm{m}$ were used as tracer particles. Each traverse line (dashed lines in Figure 1) was divided into 65 points that were unevenly distributed with a higher point density near the walls. Each point was sampled for 30 seconds. A traverse system with a resolution of $12.5 \mu \mathrm{m}$, was used to move the measuring volume. The integral time scale was estimated from autocorrelation to be $\sim 80 \mathrm{~ms}$. The sample time therefore corresponds to 180-200 uncorrelated samples giving a variability on the axial mean velocity of around $2 \%$. This variability is lower than the total uncertainties discussed in section 2.4.

Fourier Transform Infrared Spectroscopy (FTIR) technique was used to measure the concentration $C$ as described by e.g. Smith [15]. The instrument was a Gasmet DX4000 with a MCT (Mercury Cadmium Telluride) detector. The measurement was conducted along the same lines as for the velocity. The lines were discretised into 13 points and at each point the mean concentration was estimated from samples taken over 60 seconds. The FTIR gave samples at $1 \mathrm{~Hz}$ with each sample being based on an evaluation of 10 spectra. The software used to collect the samples and analyse the data was Calcmet version 4.48. Some of the measurements were validated with the use of the algorithm described by Westlye [16], and this was use to estimate an error of $4 \%$ on the measured concentration. The probe was traversed manually with an estimated uncertainty on the position of $2 \mathrm{~mm}$.

\subsection{Experimental Results}

The measured mean velocities are shown in Figure 2. They have been normalised with the actual mean bulk velocity in the pipe based on the volume flow from the orifice plate flow meter. The velocity profiles are not perfectly symmetrical around the center line. The vertical profiles are influenced by the injection pipe. This is also seen in the numerical simulations as discussed in section 4.3. Other deviations from symmetry and deviation between measurements at different Reynolds number are comparable to the variability of $2 \%$. The velocity profiles are therefore reasonably independent of Reynolds number.

The concentration profiles are shown in Figure 3 and have been normalised with mean bulk concentration estimated from the injection rate of methane divided by the flow rate of air $\left(\overline{C_{Q}}=69 \mathrm{ppm}\right.$ or $\overline{C_{Q}}=35 \mathrm{ppm}$ for $\operatorname{Re}_{D}=50000$ 

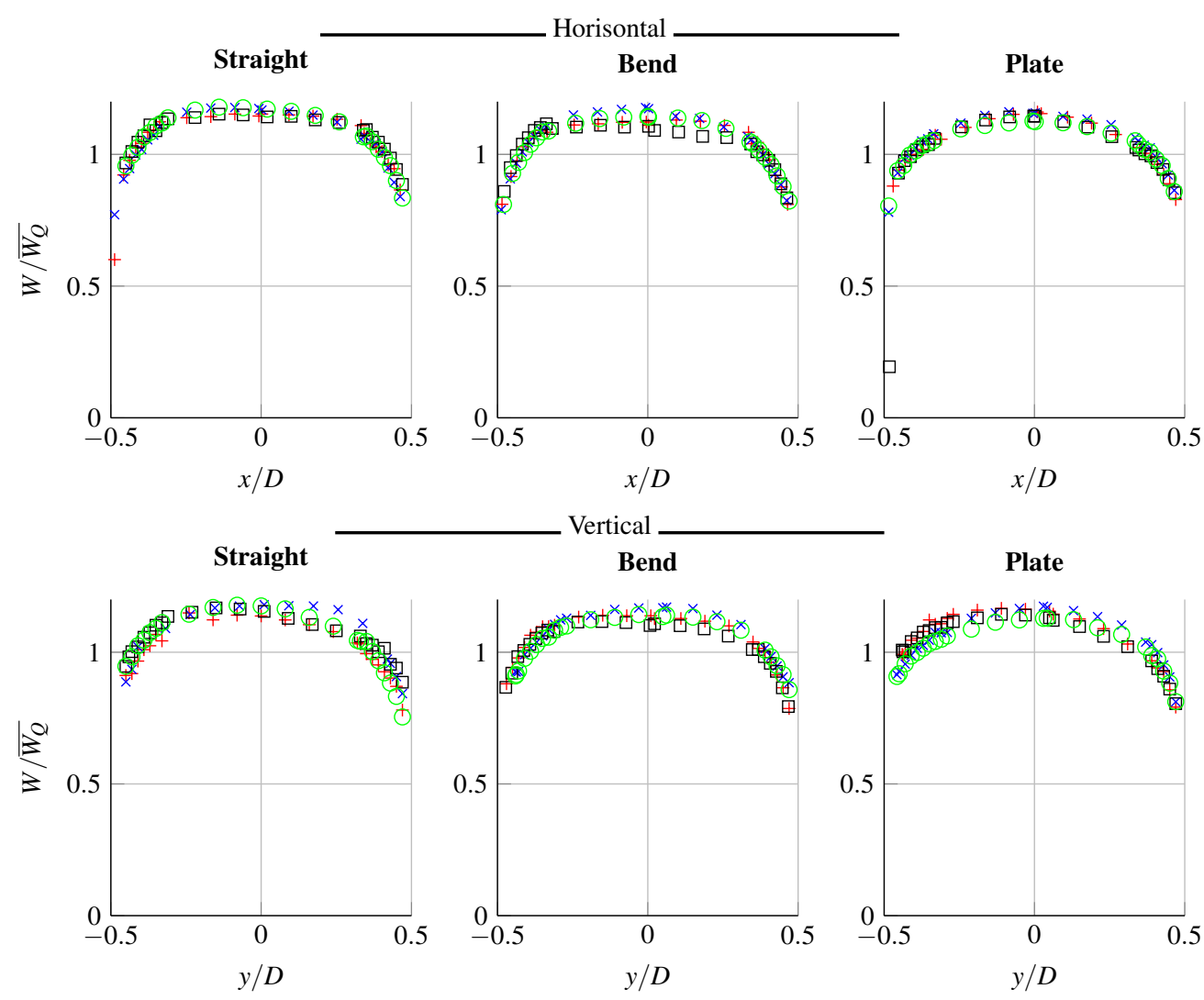

Fig. 2 The experimental obtained velocity profiles normalized with the actual mean velocity for the different positions: $(+) 05 \mathrm{D}$ and $(\times)$ 10D with $\operatorname{Re}_{D}=50000,(\square)$ 05D and (॰) 10D with $\operatorname{Re}_{D}=100000$

and $\operatorname{Re}_{D}=100000$, respectively). Looking first at the concentration profiles for the Straight and Bend configurations, a clear change is seen between profiles at 5D and at 10D with peak concentration being lower at 10D. For $\operatorname{Re}_{D}=50000$ the concentration centre peak value decrease by around $35 \%$ and for $\mathrm{Re}_{D}=100000$ by around $50 \%$. In both cases the concentration profiles becomes more flat at 10D compared to $5 \mathrm{D}$ and a plateau is created in the middle of the pipe.

For the 05DV case for the Bend, especially at the low Reynolds number, the point to point variation is larger than the general estimated uncertainty of $5 \%$, discussed in section 2.4. This is probably caused by the flow having large scale flow variation and could be reduced by longer sampling times. Increasing the Reynolds number clearly enhances mixing as seen by a reduction in peak concentration of around $10-20 \%$ when comparing $\operatorname{Re}_{D}=100000$ with $\operatorname{Re}_{D}=50000$. The measured concentration profiles are clearly not similar for $\operatorname{Re}_{D}=50000$ and $\operatorname{Re}_{D}=100000$. The concentration profiles are therefore much more sensitive to the Reynolds number than the velocity profiles.

Looking at the concentration profile with the mixer plate included, it is seen that the flow is much more mixed compared to the two other cases with an almost uniform concentration in the horizontal direction and an almost linear variation in the vertical direction with highest concentration
Table 1 Concentration at fully mixed flow conditions measured before and after the measurement campaign and compared to calculations of estimate found flow rate measurements.

\begin{tabular}{l|c|c} 
& Before & After \\
\hline Calculated [ppm] & 36.0 & 36.0 \\
Measured [ppm] & 35.2 & 37.2 \\
\hline Deviation [\%] & 2.2 & 3.3
\end{tabular}

at the top of the pipe. The concentration level is below one for nearly all the measured points indicating that an asymmetry is present. As discussed later in section 4.3, a large part of the methane has moved upwards (positive $y$ values) in the pipe.

\subsection{Uncertainty}

To validate the measurement chain for the concentration, the concentration was measured downstream of the orifice plate flow meter, but before the fan. Here the flow can be assumed to be fully mixed. This was verified by measurements at several different radial positions. The results are shown in Table 1 together with the calculated value and the deviation.

The estimated uncertainties were determined based on the fluctuations of the pressure reading over the orifice, the 

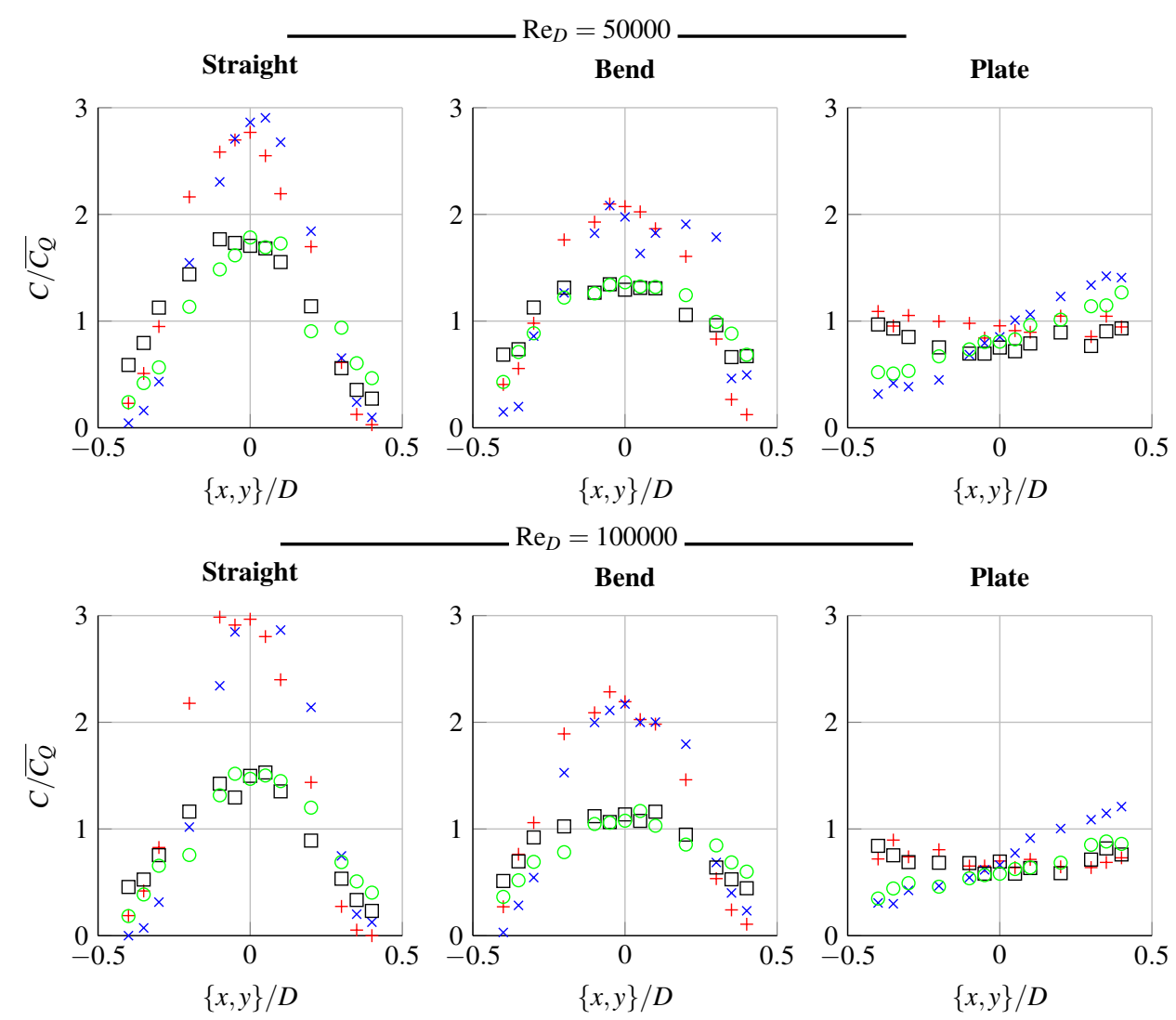

Fig. 3 The experimental obtained concentration profiles normalized with the actual mean concentration for the different positions: $(+) 05 \mathrm{DH},(\times)$ 05DV, ( $\square$ ) $10 \mathrm{DH}$ and (॰) $10 \mathrm{DV}$ for $\mathrm{Re}_{D}=50000$ and $\mathrm{Re}_{D}=100000$.

Table 2 The estimated uncertainty on the three main settings. Used to calculate the expected error. For the volume flow of $\mathrm{CH}_{4}$ the value is from the calibration certificate provide by the manufacturer of the flow regulator.

\begin{tabular}{l|c} 
Error source & Estimated uncertainty [\%] \\
\hline Volume flow air & 3.0 \\
Volume flow $\mathrm{CH}_{4}$ & 0.72 \\
FTIR - Calcmet & 4.0
\end{tabular}

specifications for the HovaGAS flow meter and the concentration value in Calcmet [16]. These are all independent values. The estimated uncertainties are given in Table 2. Based on these values, the total uncertainty was calculated to be $5 \%$. The observed deviation in Table 1 is therefore well within the estimated uncertainty of $5 \%$.

An uncertainty not directly handled is on the cross sectional area of the main pipe. Due to ovality of this pipe is the diameter varies with $D=200 \mathrm{~mm} \pm 2 \mathrm{~mm}$. This uncertainty is expected to be insignificant compared to other uncertainties.

\section{Numerical method}

\subsection{Model geometry}

The three different flow configurations described in Section 2 were each simulated with CFD. The model included 20D of straight pipe downstream of the methane injection point and all of the upstream geometry including the methane injection pipe. A sphere was added to the inlet of the pipe to facilitate the creation of the vena contracta, which is expected to be the dominant flow structure at the pipe inlet. Some details are shown for the Bend case in Figure 4.

For the injection pipe, only the last four inner pipe diameters were resolved to define the inner geometry. This length was assumed to be sufficient to facilitate the correct dynamic behaviour as pure methane enters the larger pipe. The secondary vortices formed by the 90 -degree bend in the inner pipe were assumed to be insignificant.

\subsection{Model physics}

The numerical model was a 3D incompressible CFD model of the pipe flow, modelled in CD-adapco StarCCM+ version 11. The two fluids, air and methane, were assumed to have 


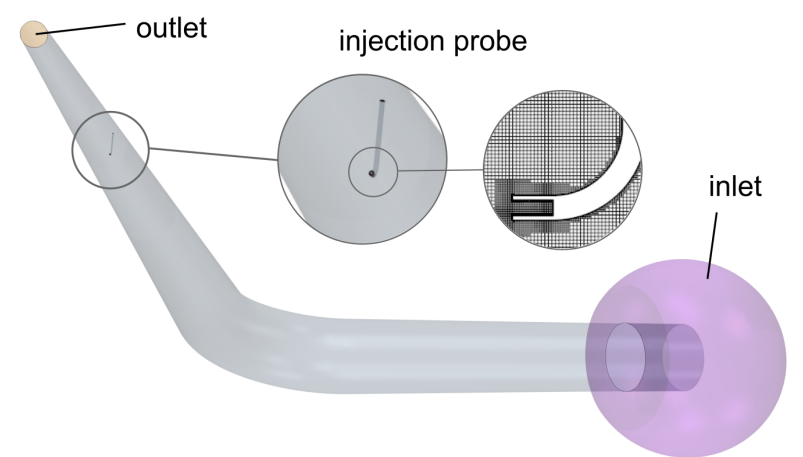

Fig. 4 Geometry in the simulation of the Bend case. The injection pipe is included in all CFD simulations.

constant density $\rho$ and dynamic viscosity $\mu$. The governing equations were solved using properties from Table 3.2 and a locally mass-weighted average of $\rho$ and $\mu$, i.e., using $\rho=$ $\rho_{\mathrm{CH}_{4}} Y+\rho_{\text {air }}(1-Y)$ and $\mu=\mu_{\mathrm{CH}_{4}} Y+\mu_{\text {air }}(1-Y)$, where $Y$ is the local mass fraction of methane.

\begin{tabular}{l|c|c|}
$i$ & $\rho_{i}\left[\mathrm{~kg} / \mathrm{m}^{3}\right]$ & $\mu_{i}[\mu \mathrm{Pa} \cdot \mathrm{s}]$ \\
\hline air & 1.184 & 18.55 \\
$\mathrm{CH}_{4}$ & 0.657 & 11.19
\end{tabular}

Table 3 Constant fluid properties for the CFD model.

Boundary conditions The sphere at the inlet was set to a stagnation inlet boundary type with a flow direction normal to the surface. Its surface area was an order of magnitude larger than the pipe cross-sectional area. This resulted in very low inlet velocities resembling the near-stagnant flow assumed to exist in the laboratory. The total pressure on this surface was uniform and constant. The turbulent intensity was fixed to $1 \%$ and the turbulent length scale was $0.2 \mathrm{~m}$. These values are assumed less important because the velocity is below $0.1 \mathrm{~m} / \mathrm{s}$ and the main turbulence contributor is the vena contracta in the pipe inlet. The pipe outlet surface was set to a uniform constant static pressure. This pressure was manually adjusted to achieve Reynolds numbers corresponding to that of the experiments. The methane inlet was set to a uniform constant velocity yielding a volume flow of methane equal to $500 \frac{\mathrm{ml}}{\mathrm{min}}$. The turbulent intensity was fixed to $1 \%$ and turbulent length scale was $0.002 \mathrm{~m}$. All walls were set to smooth walls with a no-slip condition.

Turbulence models Three different turbulence models are used. The first two, k-epsilon [17, 18] and k-omega SST [19, 20], are both widely used in the industry and belong to the family of RANS models. The third type is a transient and scale-resolving model, Spalart-Allmeras Detached Eddy (DES) $[17,18]$. DES uses Large Eddy Simulation (LES) in the main part of the flow and RANS near walls. DES is therefore computational less expensive than a full LES simulation. LES is assumed to be able to simulate directly how large scale flow motion mixes the injected gas into the air flow. Since this happens in the main flow region, it is assumed that DES will simulate this with almost the same level of details as a full LES.

In StarCCM+ a unified turbulence modelling approach was used, where the modelled momentum equation is

$\frac{\partial}{\partial t}(\rho \hat{\mathbf{v}})+\nabla \cdot(\rho \hat{\mathbf{v}} \otimes \hat{\mathbf{v}})=-\nabla \cdot \hat{p} \mathbf{I}+\nabla \cdot\left(\mathbf{T}+\mathbf{T}_{t}^{\text {model }}\right)$

where $\rho$ is fluid density in $\mathrm{kg} / \mathrm{m}^{3}, \mathbf{v}$ is velocity vector in $\mathrm{m} / \mathrm{s}$, $p$ is pressure in $\mathrm{Pa}, \mathbf{I}$ is the identity tensor, $\mathbf{T}$ is the viscous stress tensor and $\mathbf{T}_{t}^{\text {model }}$ is the modelled Reynolds stress tensor. The hat above a variable indicates either a RANS time averaged quantity or LES spatial filtered velocity [21].

The Boussinesq approximation was used to introduce a turbulent dynamic viscosity $\mu_{t}$ that relates the mean strain rate tensor $\mathbf{S}$ to the Reynolds stresses in the following way

$\mathbf{T}_{t}^{\text {model }}=2 \mu_{t} \mathbf{S}-\frac{2}{3}\left(\mu_{t} \nabla \cdot \hat{\mathbf{v}}+\rho k\right) \mathbf{I}$

where $k$ is the turbulence kinetic energy. Since the magnitude of the modelled Reynolds stress tensor scales proportionally to $\mu_{t}, \mu_{t}$ can be used as a crude measure of how much the turbulence model is attenuating the flow.

The solved transport equation for air and methane is

$\frac{\partial}{\partial t}(\rho Y)+\nabla \cdot(\rho \hat{\mathbf{v}} Y)=\nabla \cdot\left(\frac{\mu}{\sigma}+\frac{\mu_{t}}{\sigma_{t}}\right) \nabla Y$

where $Y$ is the mass fraction of methane. The Schmidt number for methane-air diffusion is $\sigma=1.0$. The turbulent Schmidt numbers $\sigma_{t}$ is a constant model parameter and the default value in StarCCM+ is $\sigma_{t}=0.9$.

No artificial resolved turbulence was applied at inlets for the DES simulations. The reason was that such turbulence would be insignificant compared to the turbulence generated by the vena contracta.

Discretization The steady RANS simulations use a secondorder upwind spatial discretisation scheme for the convective fluxes. The DES simulations uses the Hybrid-Bounded Central discretization scheme, which is the default for DES simulations in StarCCM+. The unsteady DES solver runs an implicit second-order scheme.

\subsection{Mesh}

The trimmed cell mesher in StarCCM+ was used to form predominantly cubic cells. Due to the bulk mesh cell size, the criteria for using a high Reynolds model of the turbulent boundary layer, $30<y^{+}<150$, would have been readily satisfied, but the cut-cell meshing approach gives a very uneven distribution of wall to cell centre distances in the 


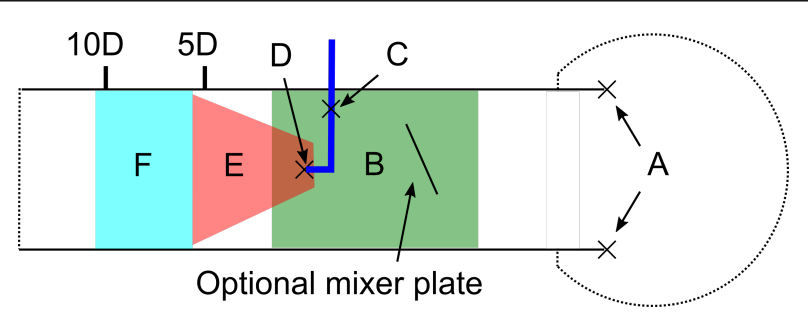

Fig. 5 Mesh topology regions for refinement purposes. Coloured patches are volume refinement regions and crosses points to specific surface features. Region B is only used when the mixer is included.

first cell layer at the walls. An uneven distribution was assumed to be detrimental to the results, despite using an all$\mathrm{y}^{+}$wall model. To achieve a smooth distribution, 5-7 layers of prismatic cells were added to the mesh near the pipe walls. The first cell height was chosen to have an average $\mathrm{y}^{+}$ below 5 as recommended for near-wall turbulence models. This study also include a sensitivity analysis of the boundary layer mesh to rationalize the boundary layer mesh setup.

The RANS and DES mesh topology consisted of volumes and surfaces where mesh was refined locally to improve the prediction of methane mixing and turbulence. The topology is illustrated in Figure 5. The surface refinement region $\mathrm{A}$ at the pipe inlet improves the resolution of the shear layer in the vena contracta. This shear layer is believed to introduce turbulence important for methane mixing. Region $\mathrm{B}$ indicates a optional volume refinement around the mixer plate, which is also a significant contributor of turbulence. A surface refinement region $C$ was added to the outside of the injection pipe wall surface to ensure a reasonable resolution of this geometry regardless of overall mesh size. For the same reason, a surface refinement region D was added at the injection pipe outlet. The methane mixing from injection and up to 10D after the methane injection point was the core region of interest. Since the inner injection pipe diameter was around $4 \mathrm{~mm}$, structures in the methane concentration of the order of $1 \mathrm{~mm}$ were expected. Resolving such structures in DES with 10 cells requires a sub-mm sized mesh. The volume refinement region $\mathrm{E}$ was necessary because such a fine mesh can only be applied in a limited region with the current numerical resources. Due to diffusion, the overall size of methane concentration structures was expected to increase downstream. Volume refinement in region $\mathrm{F}$ allowed for a gradual increase in cell size to follow that trend.

A trimmed-cell mesh only allows for 1:2 changes in cell size and 1:8 in cell volume. To minimize the numerical impact of this abrupt change in cell size a very slow growth rate is generally employed. This setting gives a minimum of 8 layers between cell size transitions. As an indirect consequence, local surface refinements will propagate into and refine the surrounding volume mesh. The applied refinement levels are seen in Table 4.
Small cells around the inlet corner in region A ensure that the starting point of the vena contracta is quite well resolved. This is not disadvantageous in terms of cell count or time step size because the affected region is relatively small and the time step is not limited by the Courant number here. The bend geometry increases the total simulation volume compared to the two other cases. To avoid a proportional increase in total cell count for the Bend configuration, the base size of the Bend DES mesh was increased. For the same reason, less surface refinement was also used at region A for the Bend case. The mesh in the inlet region for the Bend geometry and for the methane injection pipe is shown in Figure 6.

\subsection{RANS Mesh sensitivity}

The sensitivity of the RANS mesh size on velocity and concentration profiles was investigated by varying the mesh size. The variations were done at $\operatorname{Re}_{D}=50000$ and consisted of changing the straight case (s) mesh sizes to $200 \%$ (c1), $50 \%$ (f1) and $25 \%$ (f2), see table 5 . For both turbulence models, the impact of changing the mesh sizes on the velocity and the RMS profiles can be seen in Figure 7. The RMS value is not available directly in RANS, but a comparable value is estimated as $R M S(W)=\sqrt{\frac{2}{3} k}$.

The mean velocity is only weakly influenced by mesh size with the peak velocity changing less than $4 \%$. The velocity RMS profile is influenced more by mesh size, but the fine grids ( $\mathrm{f} 1$ and $\mathrm{f} 2$ ) are in good agreement, and the grid used in further simulations (s) only has small deviations from the fine grids. Irrespectively of the amount of mesh refinement, the k- $\omega$ SST does not approach the measured data. The error is a too steep mean velocity profile near the center and too high levels of RMS value with maxima near $x / D= \pm 0.2$. It is surprising that the $\mathrm{k}-\omega \mathrm{SST}$ model is not predicting this flow well, since the model has been adjusted for pipe flows [19]. However, the flow is not fully developed at this position $15 \mathrm{D}$ downstream of the inlet and is still influenced by the turbulence created in the shear layers of the flow separation related to the vena contracta at the pipe inlet. The k- $\omega$ SST model appear to overpredict the turbulence created at the vena contracta. The too high levels of turbulence in the region away from the wall distorts the mean velocity profile. The mean and RMS profiles of the $\mathrm{k}-\varepsilon$ model match much better with the measurements. Not much fidelity is gained by mesh refinement below the mesh s level.

Hence the mesh sizes, $\mathrm{f1}$, in table 5 , the boundary layer meshing strategy and the k- $\varepsilon$ turbulence model were deemed most trustworthy. 
Table 4 Characteristic length for mesh base size ans refinement zones A-F in $\mathrm{mm}$ for the three simulations, see Figure 5.

\begin{tabular}{|c|c|c|c|c|c|c|c|c|}
\hline \multicolumn{9}{|c|}{ RANS - Mesh parameters } \\
\hline Geometry & Total \# of cells & Base size & A & B & $\mathrm{C}$ & $\mathrm{D}$ & $\mathrm{E}$ & $\mathrm{F}$ \\
\hline Straight & $8.2 \cdot 10^{6}$ & 10 & 2.5 & 10 & 0.6 & 0.6 & 0.6 & 2.5 \\
\hline Bend & $8.5 \cdot 10^{6}$ & 17 & 0.9 & 3.8 & 0.5 & 0.5 & 0.5 & 7.1 \\
\hline Plate & $14 \cdot 10^{6}$ & 10 & 2.5 & 2.5 & 0.5 & 0.6 & 0.6 & 5.0 \\
\hline \multicolumn{9}{|c|}{ DES - model Mesh parameters } \\
\hline Geometry & Total \# of cells & Base size & A & B & $\mathrm{C}$ & $\mathrm{D}$ & $\mathrm{E}$ & $\mathrm{F}$ \\
\hline Straight & $69 \cdot 10^{6}$ & 5.0 & 0.2 & 5.0 & 0.5 & 0.6 & 0.6 & 1.3 \\
\hline Bend & $53 \cdot 10^{6}$ & 7.1 & 0.9 & 3.6 & 0.5 & 0.5 & 0.9 & 1.7 \\
\hline Plate & $81 \cdot 10^{6}$ & 5.0 & 0.2 & 2.5 & 0.5 & 0.3 & 0.6 & 1.3 \\
\hline
\end{tabular}
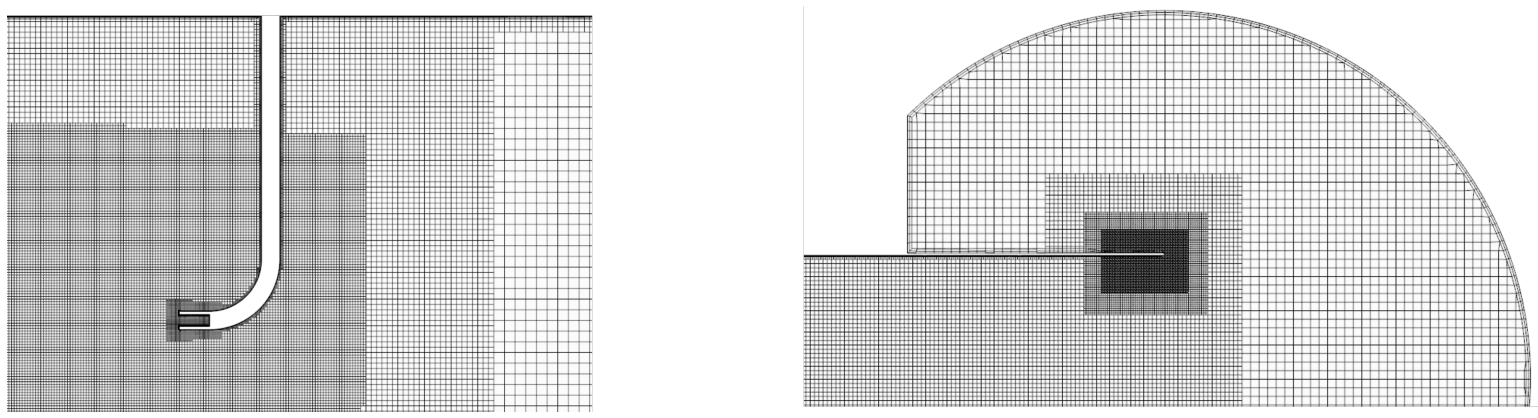

Fig. 6 Cross section showing the local mesh refinements for a region around the injection pipe (left) and at the pipe inlet (right) for the Bend case.

Table 5 Parameters for mesh refinement with zones A-F from Figure 5 in mm.

\begin{tabular}{|c|c|c|c|c|c|c|c|c|}
\hline \multicolumn{9}{|c|}{ RANS - Mesh parameters } \\
\hline Mesh & Total \# of cells & Basesize & A & B & $\mathrm{C}$ & $\mathrm{D}$ & E & $\mathrm{F}$ \\
\hline $\mathrm{c} 1$ & $1.8 \cdot 10^{6}$ & 20 & 5.0 & 20 & 0.6 & 1.3 & 1.3 & 5.0 \\
\hline $\mathrm{s}$ & $8.2 \cdot 10^{6}$ & 10 & 2.5 & 10 & 0.6 & 0.6 & 0.6 & 2.5 \\
\hline f1 & $41 \cdot 10^{6}$ & 5.0 & 1.3 & 5.0 & 0.6 & 0.3 & 0.3 & 1.3 \\
\hline $\mathrm{f} 2$ & $249 \cdot 10^{6}$ & 2.5 & 0.6 & 2.5 & 0.6 & 0.2 & 0.2 & 0.6 \\
\hline \multicolumn{9}{|c|}{ DES - Mesh parameters } \\
\hline Tag & Total \# of cells & Basesize & A & B & $\mathrm{C}$ & $\mathrm{D}$ & E & $\mathrm{F}$ \\
\hline$i$ & $0.5 \cdot 10^{6}$ & 10 & 10 & 2.5 & 2.0 & 0.6 & 10 & 10 \\
\hline$i i$ & $9.3 \cdot 10^{6}$ & 10 & 0.3 & 2.5 & 1.0 & 0.6 & 2.5 & 5.0 \\
\hline$i i i$ & $16 \cdot 10^{6}$ & 10 & 0.3 & 2.5 & 0.5 & 0.6 & 1.3 & 2.5 \\
\hline$i v$ & $69 \cdot 10^{6}$ & 5.0 & 0.2 & 5 & 0.5 & 0.6 & 0.6 & 1.3 \\
\hline
\end{tabular}

\subsection{DES mesh sensitivity}

The DES mesh size sensitivity was investigated in a short parameter study where the base size and refinement levels were changed. Due to limited computational resources it was not possible to perform a more thorough investigation. However, it demonstrated the impact of mesh size. The computational effort increased from a day in the smallest case to several months. In both cases 192 cores on nodes with dual Xeon E5-2690v3 were used. The Straight case at $\operatorname{Re}_{D}=50000$ was used as test case and the parameter variations can be seen in Table 5. The influence of mesh parameters on velocity and concentration profiles is shown in Figure 8.

The meshing parameter sets $i i, i i i$ and $i v$ gives similar concentration profiles but $i$ is very different and somewhat similar to the RANS results shown later. The same conclu- sion can be drawn from examining the axial mean flow profiles and the profiles of the RMS of axial velocity in Figure 8. At a distance of D/4 from the walls, the measured RMS values start to increase steeply towards the wall. Very near the wall, mesh ii, iii and $i v$ show a reduction in RMS level like the measurements. Mesh $i v$ is following the measured trend in RMS most closely and is therefore the mesh size needed for high fidelity CFD mixing simulations. Sufficient resolved turbulence in the CFD simulations is a key requirement for getting accurate mixing prediction. As will be demonstrated later, it is not always possible to compensate lack of resolved turbulence by parameter tuning. 


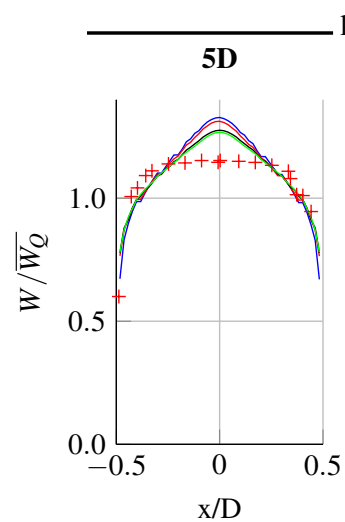

$\mathrm{k}-\omega \mathrm{SST}$

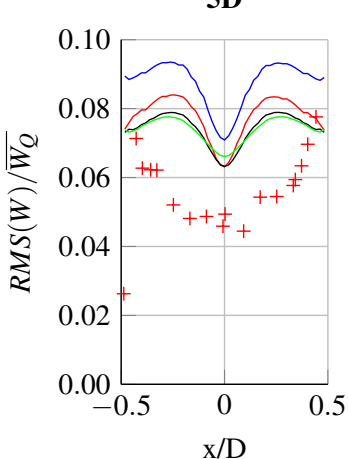

5D

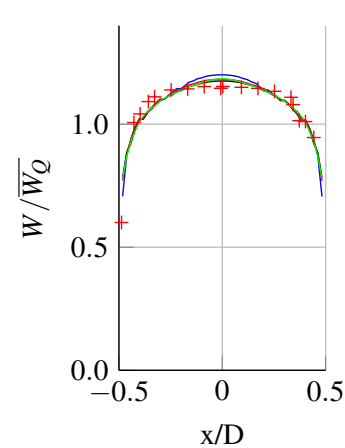

5D

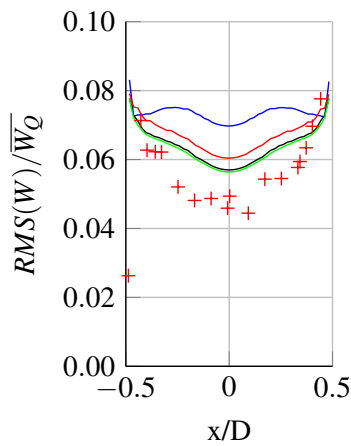

Fig. 7 Results with the Straight geometry with $\operatorname{Re}_{D}=50000$ at $z=5 \mathrm{D}$ for the horisontal case 05DH. The measured results are shown as points $(+)$. The simulated results are shown as lines, following the naming defined in Table 5. Red line is s (-), blue line is c1 (-), black line is f1 (-), Green line is $\mathrm{f} 2(-)$.
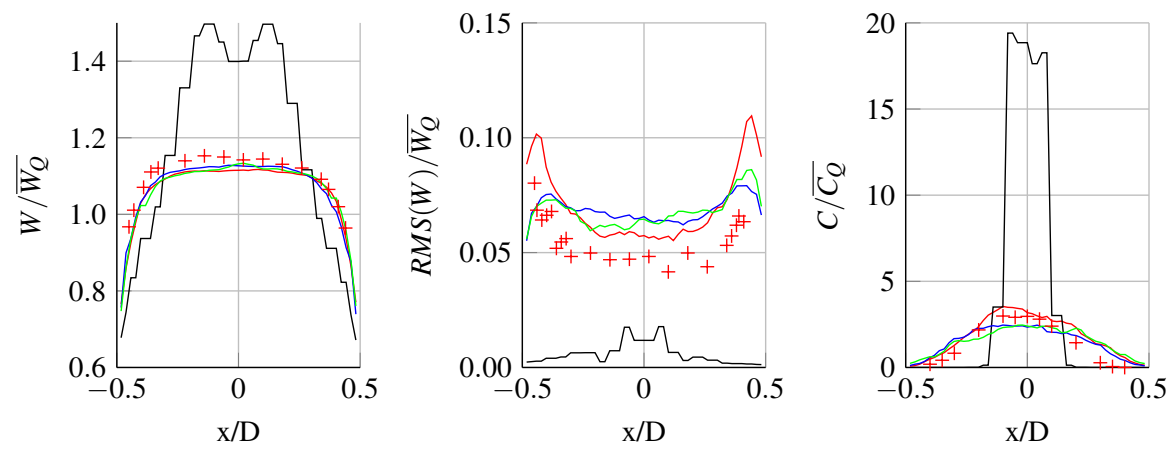

Fig. 8 DES Normalized results for the straight case with $\mathrm{Re}_{D}=50000$ at $05 \mathrm{DH}$. The experimental results are shown as (+) 05DH, the different DES mesh cases from table 5 are shown as: $\mathrm{i}(-)$, ii (-), iii (-), iv (-).

The surface uniformity $\gamma$ of a scalar $\phi$, like methane concentration, is defined in [22] and [23] as

$\gamma \equiv 1-\frac{\sum_{f}\left|\phi_{f}-\bar{\phi}\right| A_{f}}{2|\bar{\phi}| \sum_{f} A_{f}}$

where $\bar{\phi}$ is the surface area average of $\phi$ and $f$ is the index to small surface elements with area $A_{f}$ and scalar value $\phi_{f}$. The uniformity $\gamma$ is a common way of quantifying mixing in a plane. The surface uniformity can be seen to be sensitive to DES mesh size in the results in table 6. As expected, a coarser mesh also increases the $\mu_{t} / \mu$.

\subsection{Influence of turbulent Schmidt number}

As shown in Eq. (3), the turbulent Schmidt number $\sigma_{t}$ plays an important role for the effective mass diffusion in mixing simulations when $\mu_{t} / \mu \gg 1$. This is the case for the RANS $\mathrm{k}-\varepsilon$ simulations but not for the DES simulations as seen in Table 7.

To choose the best value for $\sigma_{t}$ in the RANS $\mathrm{k}-\varepsilon$ simulations, the shape of concentration profiles and a dimensionless surface uniformity were considered. It was not possible
Table 6 Impact of mesh parameters on surface uniformity $(\gamma$ from eq. (4)) evaluated at $z=5 D$ and at $z=10 D$. The volume average turbulent viscosity ratio $\mu_{t} / \mu$ is evaluated downstream of methane injection for the DES simulations. The averaging region for $\mu_{t} / \mu$ is a box centered on the pipe axis. The box extents $2 \mathrm{D}$ downstream of the methane pipe and has a width of $\mathrm{D} / 2$.

\begin{tabular}{lccc} 
Mesh & $\mu_{t} / \mu$ & $\gamma$ at $5 \mathrm{D}$ & $\gamma$ at 10D \\
\hline$i$ & 3.6 & 0.08 & 0.14 \\
$i i$ & 2.1 & 0.69 & 0.85 \\
$i i i$ & 1.9 & 0.65 & 0.81 \\
$i v$ & 0.2 & 0.64 & 0.86
\end{tabular}

Table 7 The volume average turbulent viscosity ratio $\mu_{t} / \mu$ downstream of methane injection for the RANS k- $\varepsilon$ and DES simulations with $\operatorname{Re}_{\mathrm{D}}=50.000$. The averaging region is the same as stated in Table 6 .

\begin{tabular}{lcc} 
Geometry & RANS $\mu_{t} / \mu$ & DES $\mu_{t} / \mu$ \\
\hline Straight & 198 & 0.2 \\
Bend & 42 & 0.2 \\
Plate & 275 & 0.8
\end{tabular}

to include validation values for surface uniformity because measurements were only taken along 2 lines. The simulated and measured concentration profiles at $z=5 D$ and $z=10 D$ are seen in Figure 9. At $z=5 D$ the RANS k- $\varepsilon$ profiles have a peak value in the middle and a decay towards the walls as for 

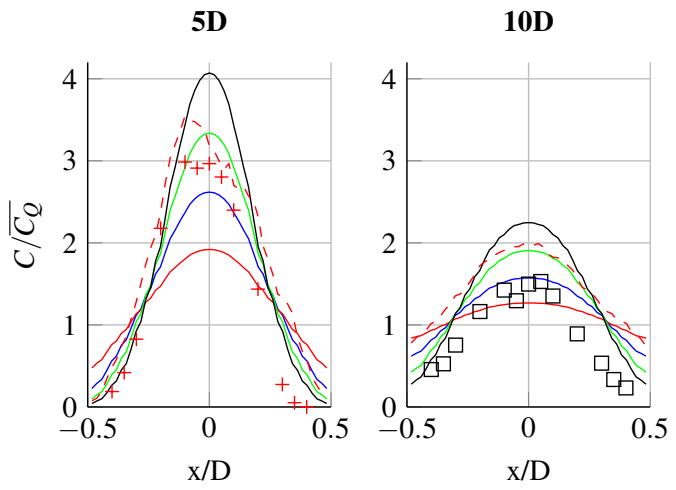

Fig. 9 Normalized concentration profiles for the straight case with $\mathrm{Re}_{D}=50.000$. The experimential results are shown with (+) $05 \mathrm{DH}$, and $(\square)$ 10DH. The impact of the turbulent Schmidt number for RANS $\mathrm{k}-\varepsilon$ model simulations are shown as $(-) \sigma_{t}=0.5,(-) \sigma_{t}=0.7,(-)$ $\sigma_{t}=0.9,(-) \sigma_{t}=1.1$. The DES simulation are shown with the dash line (- -).

Table 8 Calculated surface uniformity ( $\gamma$ from eq. (4)) at 5D and 10D for DES and RANS k- $\varepsilon$ simulations with different $\sigma_{t}$.

\begin{tabular}{lccc} 
Model & $\sigma_{t}$ & $\gamma$ at 5D & $\gamma$ at 10D \\
\hline DES & 0.7 & 0.64 & 0.86 \\
RANS & 0.5 & 0.79 & 0.91 \\
RANS & 0.7 & 0.67 & 0.84 \\
RANS & 0.9 & 0.59 & 0.77 \\
RANS & 1.1 & 0.52 & 0.70
\end{tabular}

the DES profiles. Based on the plots at $z=5 D$ the optimal value of $\sigma_{t}$ in RANS k- $\varepsilon$ simulations would be between 0.7 and 0.9. Further downstream at $z=10 D$ the optimal value of $\sigma_{t}$ seems to be about 0.7 . At $z=10 D$ the simulated curves are too flat.

In Table 8 , the impact of $\sigma_{t}$ on surface uniformity 5D and 10D downstream of injection point is given. As should be expected, a more flat profile leads to higher surface uniformity. To match the DES results at 5D the optimal value of $\sigma_{t}$ is between 0.7 and 0.9 but at 10D it should be between 0.5 and 0.7 . Given the ambiguity in the optimal value for $\sigma_{t}$, it was decided to use $\sigma_{t}=0.7$. This value was a compromise between the values for the different downstream locations (5D and 10D), at least for a Straight case.

\section{Results and Discussions}

\subsection{RANS k- $\varepsilon$ simulations}

Based on the RANS turbulence model validation show in section 3.4, it is chosen only to show results obtained with the RANS k- $\varepsilon$ model. A comparison between measured and calculated velocities and concentration profiles for all three cases at a Reynolds number of 50000 and 100000 is shown in Figure 10.

The measured profiles of axial velocity are quite similar for the three geometries irrespectively of Reynolds number.
In contrast, the RANS results show significant differences between the three profiles. While the Straight configuration is in good agreement with measurements, the Plate configuration has too steep velocity gradients in the inner part of the pipe and the Bend configuration has a strongly skewed profile not seen in the measured profiles. The RANS results for concentration are in good agreement with measurements for the Straight and Plate configurations, but is completely wrong for the Bend configuration. Here, RANS predict all methane to go one side of the pipe while the measurements show a symmetric distribution. There is only modest changes with Reynolds numbers for RANS.

The differences in velocity profile shape are likely to be caused by an underprediction of turbulent momentum diffusion in RANS as discussed in section 3.4. In general this leads to steeper velocity profiles in RANS compared to measurements. A bend creates a secondary flow structure downstream of the bend that consists of two counter-rotating vortices. In the RANS simulations for the Bend case, the peak in axial velocity was still clearly off-axis at $5 \mathrm{D}$, in contrast to the measurements. This indicates that the secondary flow structure persist for too long time. This is again likely to be a result of insufficient momentum mixing in RANS. Prediction of concentration depend both on convection by the flow and by turbulent diffusion. The turbulent diffusion is in RANS directly linked to momentum diffusion through the turbulent viscosity. A too low concentration diffusion linked with an over predicted secondary flow can therefore explain the completely wrong concentration distribution predicted by RANS.

\subsection{DES}

The DES results shown in Figure 11 show much better agreement with measurements than the RANS simulations. Compared to the experiments, the DES velocity profiles for the Straight and Bend configurations have a more flat profile away from the walls and a slightly higher velocity near the wall. For the Plate configuration, the DES velocities agree very well with experiment. The DES results are very similar to the measurements in terms of RMS. The simulated and measured velocity profiles do not have up-down symmetry because of the methane injection pipe. This is discussed in section 4.3 .

The concentration from both experiments and DES show random variation from point to point. The averaging caused by the $1 \mathrm{~Hz}$ data rate for the concentration measurements results in a lower observed RMS value (not shown) than an instantaneous measurement would give. The actual flow have quite high temporal variation due to discrete blobs of methane being convected while slowly mixing. This is illustrated in a DES snapshot in Figure 12. The blobs cause a larger variability in the measured concentration than seen 

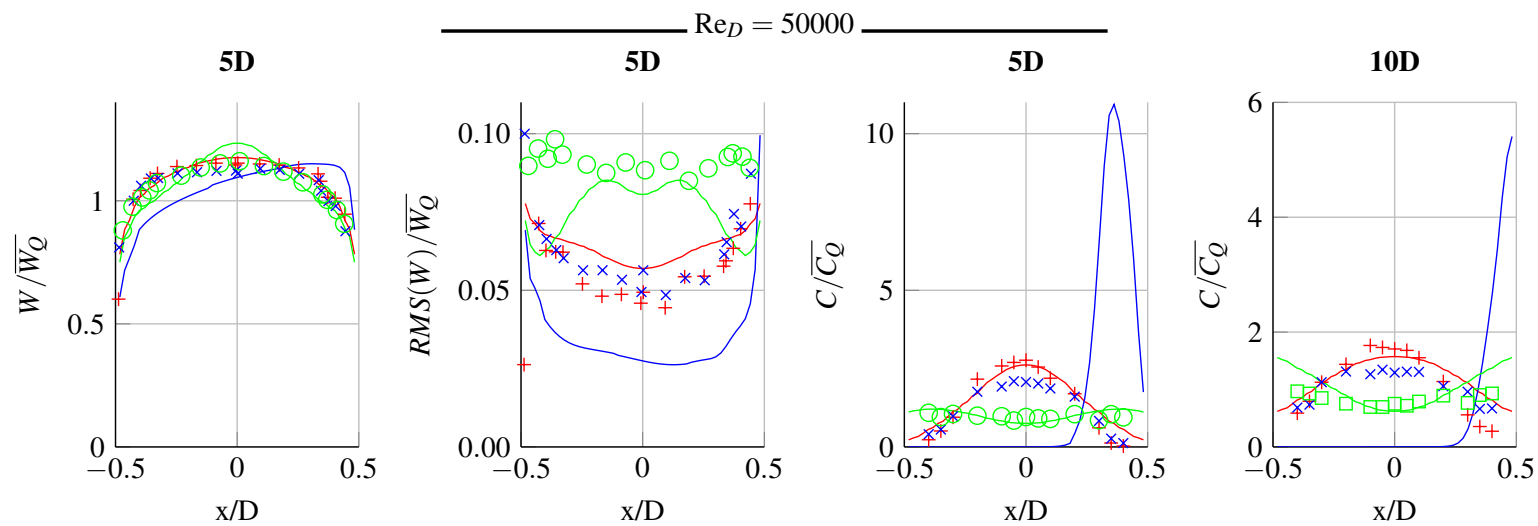

5D

5D

5D

10D
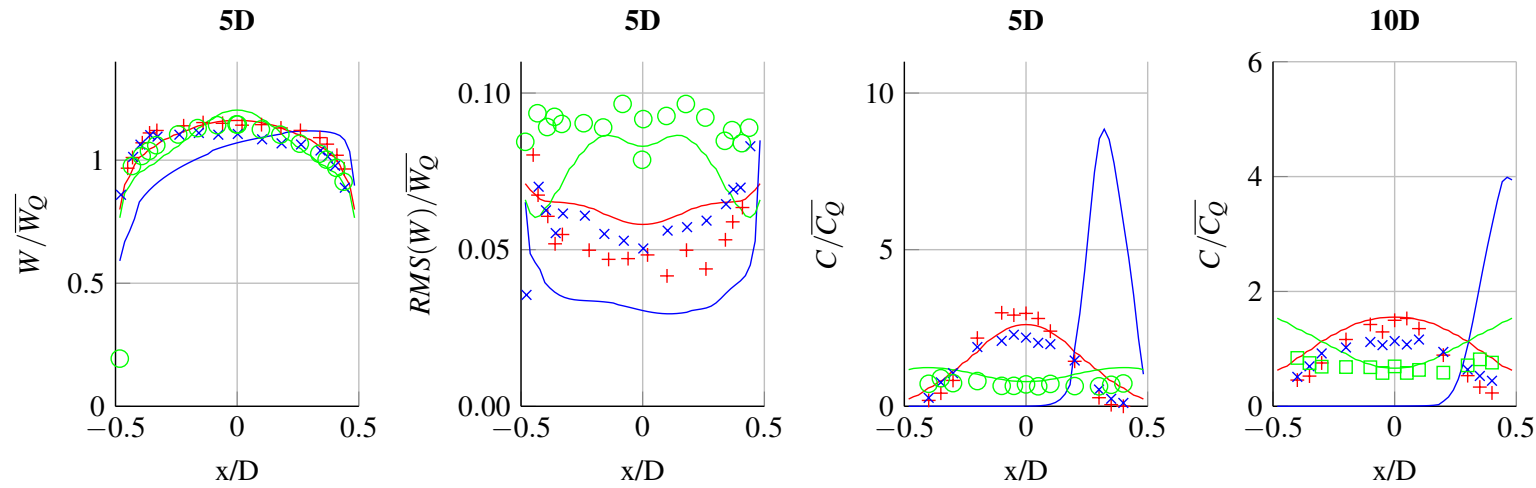

Fig. 10 RANS and experiments at a $\operatorname{Re}_{D}=50000$ and $\operatorname{Re}_{D}=100000$ for the horisontal results. The measured results are shown as points and the simulated as a line: Straight $(+,-)$, Bend $(\times,-)$, Plate $(\circ,-)$.

for velocity. The variability in the DES results are also relatively high due to the practical limitation in simulation time. The simulation time corresponds to approximately 60 integral time scales where the integral time scale is estimated from autocorrelation of velocity time series at different monitor points. The statistical variability on the mean velocity is in the order of $2 \%$. As the concentration has much higher fluctuations, the statistical error on the mean concentration is in the order of $15 \%$. The asymmetries in the mean concentration in Figure 11 can be explained by this variability. However, the variability is low enough to show fair agreement between experiment and DES results for most cases.

For $\operatorname{Re}_{\mathrm{D}}=100000$ and $z=10 D$, the DES results have higher level of the concentration than the experiments for the Plate configuration. The methane is clearly being displaced toward the upper side of the pipe as also seen in Figure 3. This is likely to be caused be secondary flow motion created by the inclined plate, where the flow moves upwards at the center of the pipe and downwards near the side walls. Selected profiles in vertical direction are shown in Figure 13. Here it is seen that the concentration is also predicted to be too high at upper side of the pipe for the Plate configuration. Small deviation in how fast the secondary motion is, could result in significant difference in concentration level in hor- izontal profiles. This secondary motion is apparently quite slow and difficult to predict.

A similar tendency is seen for the Straight configuration, where concentration in Figures 11 and 13 is estimated by DES to be too high compared to the experiments. This could be explain by a similar up-down asymmetry discussed in the next section.

\subsection{Asymmetry}

An up-down asymmetry is observed in the experiments, e.g. in Figure 2. The injection pipe retards the flow at the top of the pipe and as a consequence the flow must accelerate at the bottom of the pipe. The methane was injected into the below-average momentum fluid and peak concentration was therefore displaced upwards. Very far downstream this up-down asymmetry must disappear, but it is still clear at 10D although these plots are not shown. Figure 13 focuses on the up-down asymmetry along the vertical traverse line 05DV. Both experiment and DES show that the peak axial velocity lies in the lower part and peak concentration lies in the upper part. Figure 14 provides a full overview of the 5D cross section from DES of the Straight case. This overview confirms the presence of a distinct up-down asymmetry. 

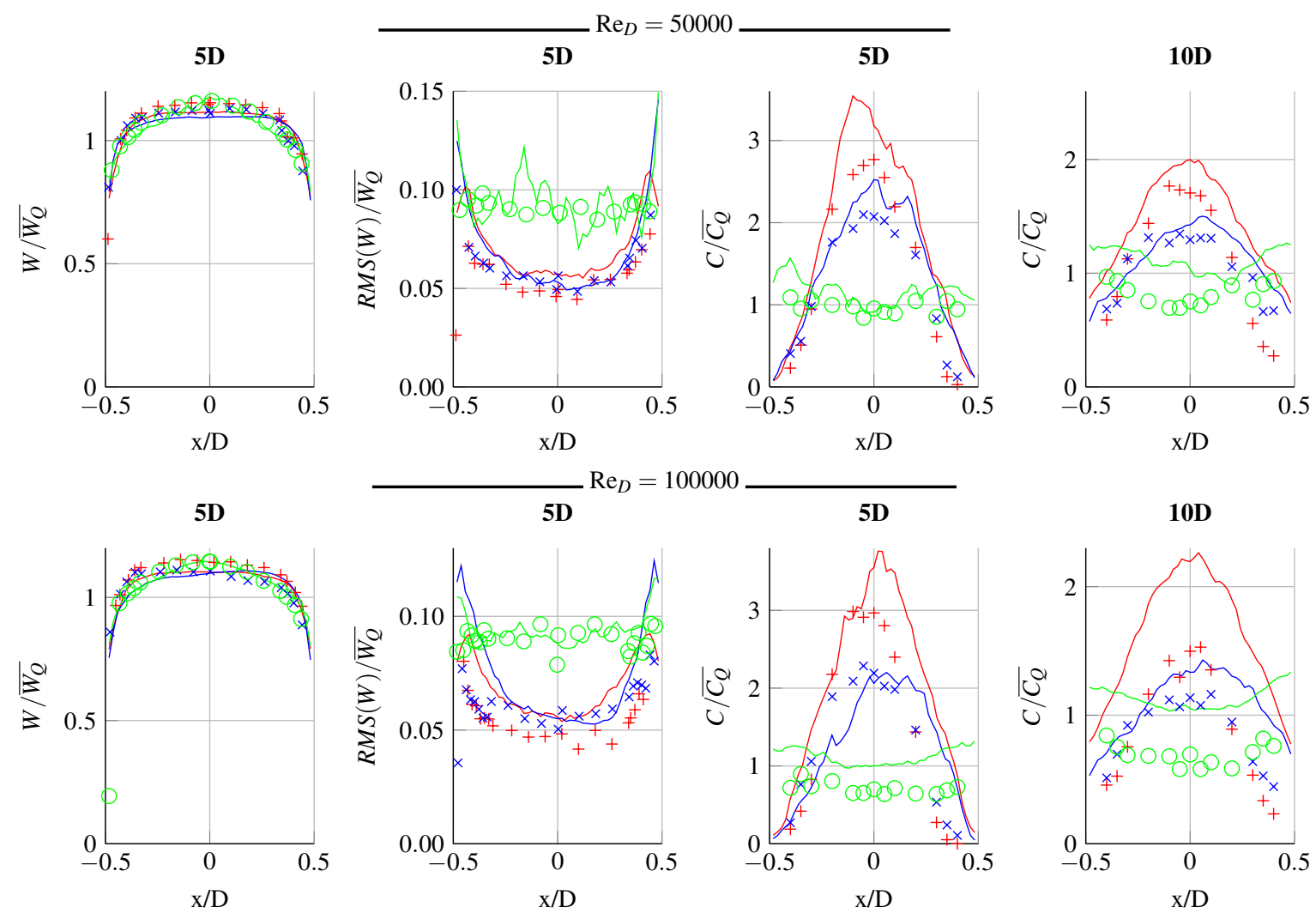

Fig. 11 DES and experiments at a $\operatorname{Re}_{D}=50000$ and $\operatorname{Re}_{D}=100000$ for the horisontal results. The measured results are shown as points and the simulated as a line: Straight $(+,-)$, Bend $(\times,-)$, Plate $(\circ,-)$.

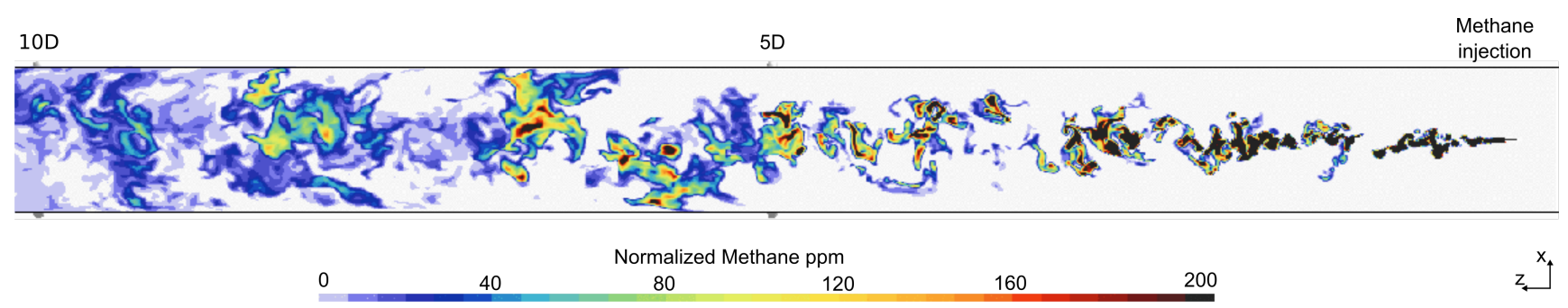

Fig. 12 DES instantaneous xz cross plane of the normalised concentration along the length of the pipe for the DES simulated Straight case at $\operatorname{Re}_{D}=50000$.

\section{Conclusion}

Mixing in pipe flow has been investigated in three geometries relevant for industrial applications: a short straight pipe, a $90^{\circ}$ bend connecting two short straight pipes and a short straight pipe with a mixer plate inserted. All short straight pipe elements were 10 diameters long and each configuration started with a sharp-edged inlet. This is in contrast to many earlier published investigations that typically use a fully developed pipe flow as inlet. Here the flow only depend on the turbulence model's interaction with fully developed near wall flow. For a sharp-edged inlet, the turbulence is started in the vena contracta near the walls at the pipe entrance. This is a more realistic condition for many practical applications.
The mean axial velocity component were measured at several vertical and horizontal lines using Laser Doppler Anemometry. A tracer gas was used for the mixing experiments. The tracer gas was injected at the pipe center with a small pipe with a $90^{\circ}$ bend. The concentration was measured along the same lines as the velocity. This data set is suitable for testing different simulation models.

Two RANS turbulence models were tested: the k- $\varepsilon$ model and the k- $\omega$ SST model. The latter had difficulties in predicting even the flow in the short straight pipe. The momentum diffusion seem to be underpredicted in the flow in the central part of the pipe resulting in a almost laminar velocity profile near the center. Only the k- $\varepsilon$ model was used for more detailed investigations. A scale resolved DES model was also 

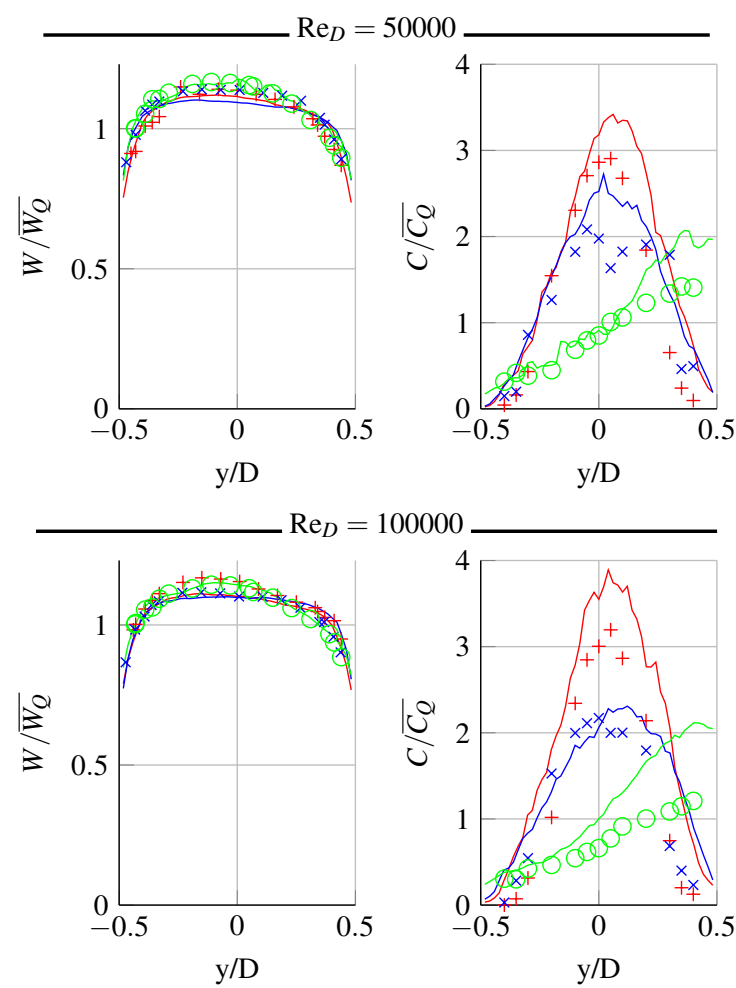

Fig. 13 DES and experiments at a $\operatorname{Re}_{D}=50000$ and $\operatorname{Re}_{D}=100000$ at 05DV. The measured results are shown as points and the simulated as a line: Straight $(+,-)$, Bend $(\times,-)$, Plate $(\circ,-)$.
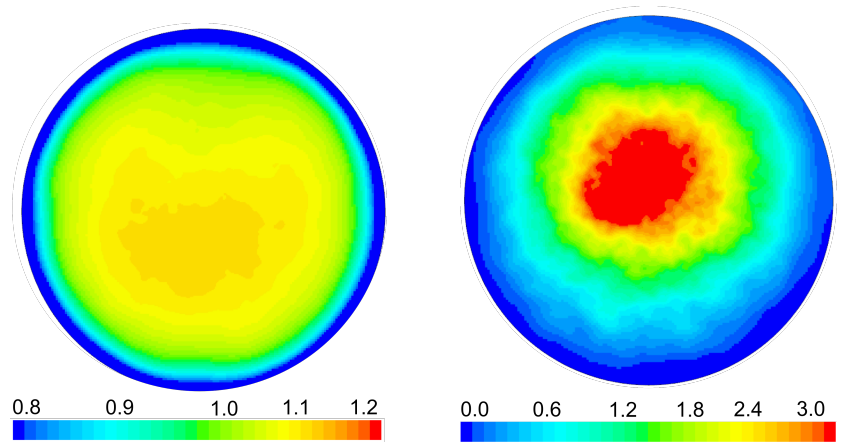

Fig. 14 DES results for the Straight case at $5 \mathrm{D}$ for $\mathrm{Re}_{D}=50000$ in a pipe cross section 5D downstream the methane injection point. Left: Normalized mean velocity, right: normalized concentration.

tested. For both type of simulations a study of grid resolution were performed.

For the k- $\varepsilon$ model, the experiment and simulation for velocity and concentration profiles were in good agreement for the short straight pipe inlet after some tuning of the turbulent Schmidt number. For the mixer plate configuration some minor deviations were found between measurements and simulations. For the Bend configuration the profiles were quantitatively and qualitatively wrong. The simulations seem to overpredict the secondary flow generated in the pipe bend and underestimate the mixing. For the DES, the velocity profiles were inside the estimated uncertainty for the exper- iments. The concentration profiles have a higher value than the experimental, but the trends are alike. The reason for the higher concentration values are likely related to how well DES captures the effect of the methane pipe on the main flow.

The k- $\varepsilon$ model therefore appears to be a reasonable model for simple pipe flow with mixing and for a flow with a mixing plate that generated strong turbulence. For more complex cases with secondary flow, the model can give completely wrong results. The DES is capable of handing the more complex flow, but at the cost of much longer simulation time. It would be interesting to optimize mesh resolution and numerical settings further to get the best trade-off between accuracy and computational speed for DES.

Acknowledgements This project was funded by the European Union Horizon 2020 - Hercules-2 - research and innovation program under Grant Agreement No. 634135.

\section{Conflict of interest}

The authors declare that they have no conflict of interest.

\section{References}

1. Reynolds O (1883) An experimental investigation of the circumstances which determine whether the motion of water shall be direct or sinuous, and of the law of resistance in parallel channels. Proceedings of the royal society of London 35(224-226):84-99

2. Taylor GI (1922) Diffusion by continuous movements. Proceedings of the london mathematical society 2(1):196-212

3. Dimotakis PE (2005) Turbulent mixing. Annu Rev Fluid Mech 37:329-356

4. Paul EL, Atiemo-Obeng VA, Kresta SM (2004) Handbook of industrial mixing: science and practice. John Wiley \& Sons

5. Jordan D (1961) A theoretical study of the diffusion of tracer gas in an airway. The Quarterly Journal of Mechanics and Applied Mathematics 14(2):203-222

6. Lee J, Brodkey RS (1964) Turbulent motion and mixing in a pipe. AIChE Journal 10(2):187-193

7. Filmer RW, Yevjevich V (1967) Experimental results of dye diffusion in large pipelines. In: Proceedings of 12th Congress of the International Association for Hydraulic Research, Colorado State University, pp 115-120

8. Evans G (1967) A study of diffusion in turbulent pipe flow. Journal of Basic Engineering 89(3):624-631

9. Clayton CG, Ball A, Spackman R (1968) Dispersion and mixing during turbulent flow of water in a circular pipe. Tech. rep., United Kingdom Atomic Energy Authority, Wantage (England). Research Group

10. Ger A, Holley E (1976) Comparison of single-point injections in pipe flow. Journal of the Hydraulics Division

11. Guiraud P, Bertrand J, Costes J (1991) Laser measurements of local velocity and concentration in a turbulent jet-stirred tubular reactor. Chemical engineering science 46(5-6):1289-1297

12. Laufer J (1954) The structure of turbulence in fully developed pipe flow. NASA reports REPORT 1174 
13. Eggels J, Unger F, Weiss M, Westerweel J, Adrian R, Friedrich R, Nieuwstadt F (1994) Fully developed turbulent pipe flow: a comparison between direct numerical simulation and experiment. Journal of Fluid Mechanics 268:175-210

14. ISO5167-2:2003(E) (2003) Measurement of fluid flow by means of pressure differential devices inserted in circular-cross section conduits running full. International Organization for Standardization

15. Smith BC (2011) Fundamentals of Fourier transform infrared spectroscopy. CRC press

16. Westlye FR (2012) Emission measurement and exhaust aftertreatment of an ammonia fueled si-engine. Master Thesis, Technical University of Denmark

17. Jones WP, Launder BE (1972) The prediction of laminarization with a two-equation model of turbulence. International Journal of Heat and Mass Transfer 15(2):301-14, 301-314, DOI 10.1016/0017-9310(72)90076-2

18. Launder BE, Sharma BI (1974) Application of the energydissipation model or turbulence to the calculation of flow near a spinning disc. Letters in Heat and Mass Transfer 1(2):131-7, 131137, DOI 10.1016/0094-4548(74)90150-7

19. Menter F (1994) Two-equation eddy-viscosity turbulence modeling for engineering applications. AIAA Journal 32(8):1598-1605

20. Wilcox D (2008) Formulation of the k-omega turbulence model revisited. AIAA Journal 46(11):2823-2838

21. CD-adapco (2017) STAR-CCM+ Manual. CD-adapco, 11th edn

22. Weltens H, Bressler H, Terres F, Neumaier H, Rammoser D (1993) Optimisation of catalytic converter gas flow distribution by cfd prediction. Tech. rep., SAE Technical Paper

23. Johansson Å, Wallin U, Karlsson M, Isaksson A, Bush P (2008) Investigation on uniformity indices used for diesel exhaust aftertreatment systems. Tech. rep., SAE Technical Paper 